\title{
THE GENERALIZED QUANTUM DATABASE SEARCH ALGORITHM
}

\author{
SÁNDOR IMRE, FERENC BALÁZS
}

\begin{abstract}
In this paper we present a generalized description of the Grover operator, employed in a quantum database search algorithm. We will discuss the relation between the original and generalized operators as well as we will show the optimal setting of parameters used in the Grover operator leading to a single iteration during a database search.
\end{abstract}

\section{INTRODUCTION}

L. K. Grover published his fast database searching algorithm first in [1] and [2] using the diffusion matrix approach to illustrate the effect of the Grover operator, that took $\mathcal{O}(\sqrt{N})$ iterations to carry out the search, which is the optimal solution, as it was proved in [3]. Boyer, Brassard, Hoyer and Tapp [4] enhanced the original algorithm for more than one marked entry in several number of identical solutions in the database and introduced upper bounds for the required number of evaluations.

After a short debate Bennett, Bernstein, Brassard and Vazirani gave the first proof of the optimality of Grover's algorithm in [5]. The proof was refined by Zalka in $[6]$.

Later the rotation in two dimensional state space (with the bases of separately superpositioned marked and unmarked states) $\mathrm{SU}(2)$ approach were introduced by Aharonov in [7]. Within this book we followed this representation form according to its popularity in the literature.

During the above mentioned evolution of the Grover algorithm a new quest started to formulate the building blocks of the algorithm as generally as possible. The motivations for putting so much effort into this direction were on one hand to get a much deeper insight into the heart of the algorithm and on the other hand to overcome the main shortcoming of the algorithm, namely the sure success of finding a marked state can not be guaranteed. In [8] the authors replaced the Hadamard transformation with an arbitrary unitary one. The next step was the introduction of arbitrary phase rotations in the Oracle and the phase shifter instead of $\pi$ in [9]. To provide sure success at the final measurement Brassard at all [10] run the original Grover algorithm, but for the final turn a special Grover operator Grover operator with smaller step was applied. Hoyer et al. [11] gave another ingenious solution of the problem. They modified the original Grover algorithm and the initial distribution.

To give another viewpoint Long at al introduced the three dimensional SO(3) picture in description of Grover operator in [12]. The achievements were summarized and extended by Long [13] and an exact matching condition was derived for

Key words and phrases. Grover's Algorithm, Quantum computing, Quantum Signal Processing, Grover database search, generalized Grover Operator. 
multiple marked states in [14]. Unfortunately the $\mathrm{SO}(3)$ picture is less picturesque and it misses the global phase factor before the measurement. In normal case it does not cause any difficulty because measurement results are immune of it. However, if it is planed (we plan) to reuse the final state of the index register without measurement as the input of a further algorithm (operator), it is crucial to deal with the global phase. Therefore, Hsieh and Li [15] returned to the traditional two dimensional $\mathrm{SU}(2)$ formulation and derived the same matching condition foe one marked element as Long achieved but they saved the final global phase factor. One important part of these solution, however, was missing. Namely, they required that the initial sate should fit into the two dimensional state space defined by the marked and unmarked states. This gives large freedom for designers but encumber the application of the generalized Grover algorithm as a building block of a larger quantum system.

Therefore another very important question within this topic proved to be the analysis of the evolution of Grover's algorithm when it is started from an arbitrary initial state, i.e. the amplitudes are either real or complex and follow any arbitrary distribution. In this case sure success can not be guaranteed, but the probability of success can be maximized. Biham and his team first gave the analysis of the original Grover algorithm in [16], [17]. In [18] the analysis was extended to the generalized Grover algorithm with arbitrary unitary transformation and phase rotations.

Within this paper we combined and enhanced the results for generalized Grover search algorithm in terms of arbitrary initial distribution, arbitrary unitary transformation, arbitrary phase rotations and arbitrary number of marked items to build a method to construct an unstructured data base search algorithm which can be included inside a quantum computing system. Because its constructive nature this algorithm is capable to get any amplitude distribution at its input, provides sure success in case of measurement and allows to connect its output to another algorithm if no measurement is performed. Of course, this approach assumes that the initial distribution is given and it determines all the other parameters according to the construction rules. However, readers who are interested in applying a predefined unitary transformation as the fixed parameter should settle for a restricted set of initial states and suggested to take a look at [15].

Grover's data base search algorithm assumes the knowledge of the number of marked states, but it is typical that we do not have this information in advance. Brassard et al. [19] gives the first valuable idea how to estimate the missing number of marked states, which was enhanced in [10] and traced back to a phase estimation of Grover operator.

A rather useful extension of the Grover algorithm when we decided to find minimum/maximum point of a cost function. Dürr and Hoyer suggested the first statistical method and bound to solve the problem in [20]. Later based on this result Ahuya and Kapoor improved the bounds in [21]. A further beneficial exertion possibilities of the Grover algorithm can be employed in Telecommunication field. The present authors introduced the Grover database search based multiuser detection in WCDMA environment in [22], [23], and [24].

Recently Grover emphasized in [25] that the number of elementary unitary operations can be reduced which lunched a new quest for the most effective Grover structure in terms of number of basic operations. 
The rest of the paper is organized as follows. In Section 2. the original Grover quantum database search operator $(\mathcal{G})$ is reviewed. In Section 4 . we introduce the generalized Grover operator $(\mathcal{Q})$ wherefor an example is shown in Section 5 . The paper is closed with a final conclusion in Section 6 .

\section{Basic Grover Algorithm}

Consider a large unsorted database, which contains $N$ entries, to find the desired value with any classical algorithms would need at least $\mathcal{O}(N)$ steps.

2.1. The Searching Algorithm. Grover's quantum searching algorithm $(\mathcal{G})$ consists of four simple operations: an Oracle followed by a Hadamard-, a controlled phase-, and a Hadamard-transformation again, as depicted in Figure 1. In the next sections we bring up the main behavior and functionality of $\mathcal{G}$, apartly.

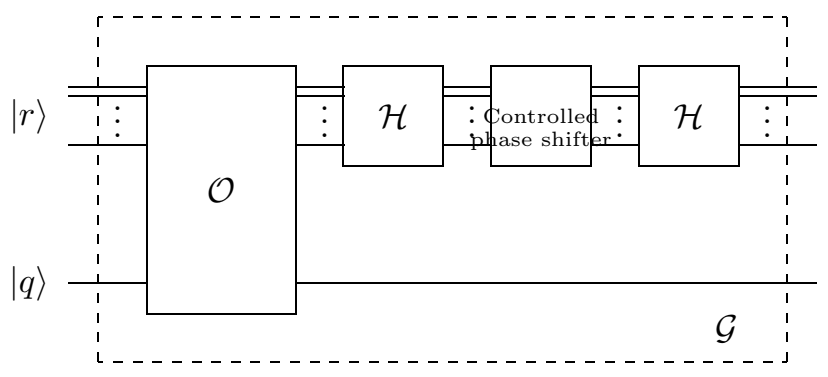

FIGURE 1. Sketch of Grover's database search circuit

2.1.1. The Oracle. Let us consider a database with $N=2^{n}$ entries, a quantum index register $|r\rangle$ containing all the index values of the database in a quantum superposition and a black box, the so called Oracle. Grover's gorgeous brainchild was to implement a binary function $f(x)$ in the Oracle, with the property

$$
f(x)= \begin{cases}1 & \begin{array}{l}
\text { if the entry belonging to index } x \text { matches } \\
\text { the searched item } \\
0 \quad \text { otherwise }
\end{array}\end{cases}
$$

$x=0,1, \ldots,(N-1)$. The function in (1) can have the value 1 either at a single or multiple values of $x$, depending on how many identical searched entries in a particular database exist. Entries, which are solutions for the search problem are called marked states according to the literature and ones which do not lead to a solution are referred as non marked states.

Invoking the Oracle $(\mathcal{O})$ with the following computation rule (see Fig. 2) :

$$
|x\rangle|q\rangle \stackrel{\mathcal{O}}{\mapsto}|x\rangle|q \otimes f(x)\rangle,
$$

where $|x\rangle_{N}$ and $|q\rangle=\left(\frac{|0\rangle-|1\rangle}{\sqrt{2}}\right)$ are the wanted $n=\operatorname{ld} N$ qubit vector basis state (i.e. the index in question) and an auxiliary one qubit state, respectively, the output 
of the oracle will act as (see also the Deutsch-Jozsa algorithm in Sec. xx.)

$$
|x\rangle\left(\frac{|0\rangle-|1\rangle}{\sqrt{2}}\right) \stackrel{\mathcal{O}}{\mapsto}\left\{\begin{array}{cl}
|x\rangle\left(\frac{|0\rangle-|1\rangle}{\sqrt{2}}\right) & \text { for } x, \text { whose entry } \\
-|x\rangle\left(\frac{|0\rangle-|1\rangle}{\sqrt{2}}\right) & \text { is not in the database } \\
& \text { for } x \text { whose entry } \\
& \text { can be found in the } \\
\text { database, }
\end{array}\right.
$$

or in more common compact mathematical form

$$
|x\rangle \stackrel{\mathcal{O}}{\rightarrow}(-1)^{f(x)}|x\rangle
$$

where $|q\rangle$ does not change during the algorithm and can be neglected henceforth.

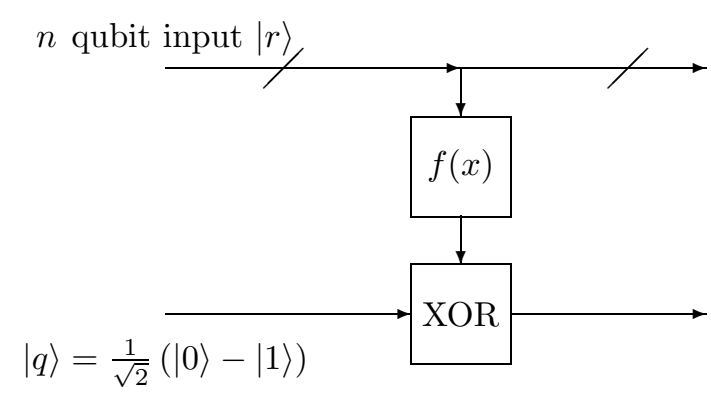

Figure 2. Inside in the Oracle $\mathcal{O}$

The input state of the index register $|r\rangle$ feeded to the Oracle is assumed to be an equal superposition state of all the possible indexes

$$
|r\rangle=|\gamma\rangle \triangleq \mathcal{H}^{\otimes^{n}}|\mathbf{0}\rangle_{n}=\frac{1}{\sqrt{N}} \sum_{x=0}^{N-1}|x\rangle
$$

where $\mathcal{H}^{\otimes^{n}}$ denotes the $n$-bit Hadamard-transformation whereas $|\mathbf{0}\rangle_{n}=|\underbrace{0 \cdots 0}_{n}\rangle$. $|\gamma\rangle$ is depicted in Fig. 3. Let $x=y$ denote the index of the marked entry, whose amplitude is drawn with thick line in the figure.

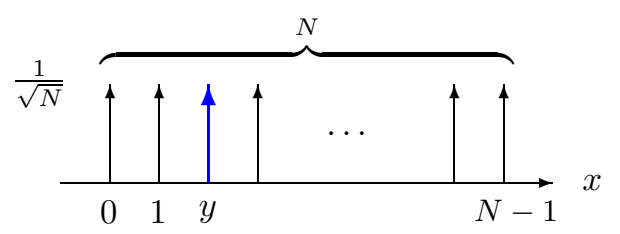

FiguRE 3. The equal superposition input state $|\gamma\rangle$

Equation (3) can be interpreted as $f(x)$ flips the sign of the amplitude of the marked state(s) and let the others unaltered (see Fig 4). One can express the 
strategy of the Oracle according to the equation (4) in operator formalism such as

$$
\mathcal{O} \triangleq \mathcal{I}-2|y\rangle\langle y|,
$$

where $(|y\rangle\langle y|)$ stands for the outer product of marked state $|y\rangle$ as well as $\mathcal{I}$ denotes here the $N$ dimensional identity matrix.

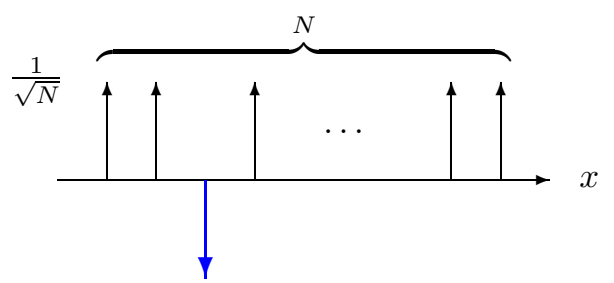

Figure 4. The input state $|\gamma\rangle$ after invoking the Oracle

As an example, let the third state be marked $(y=2)$ in an $n=2$ qubit quantum index register, i.e. $|y\rangle=\left[\begin{array}{llll}0 & 0 & 1 & 0\end{array}\right]^{T}$. Substituting $|y\rangle$ into (5) the matrix $\mathbf{O}_{y}$ representing the Oracle can be calculated as

$$
\begin{aligned}
\mathbf{O}_{y=2} & =\mathcal{I}-2\left[\begin{array}{l}
0 \\
0 \\
1 \\
0
\end{array}\right]\left[\begin{array}{llll}
0 & 0 & 1 & 0
\end{array}\right]=\mathcal{I}-2\left[\begin{array}{llll}
0 & 0 & 0 & 0 \\
0 & 0 & 0 & 0 \\
0 & 0 & 1 & 0 \\
0 & 0 & 0 & 0
\end{array}\right] \\
& =\left[\begin{array}{cccc}
1 & 0 & 0 & 0 \\
0 & 1 & 0 & 0 \\
0 & 0 & -1 & 0 \\
0 & 0 & 0 & 1
\end{array}\right]
\end{aligned}
$$

which corresponds to the amplitude flip depicted in Fig. 5.

2.1.2. Another phase reflection - Inversion about the average. The Oracle followed first by an $n$-dimensional Hadamard-gate $(\mathcal{H})$, whose output qubits feed a Controlled Phase Shifter $(\mathcal{P})$, defined as

$$
\mathcal{P} \triangleq(2|\mathbf{0}\rangle\langle\mathbf{0}|-\mathcal{I}) .
$$

$\mathcal{P}$ changes the sign of all computational basis states except for $x=0$. The final operation is again a Hadamard-gate $(\mathcal{H})$. The Hadamard-gates and the controlled phase shifter inverts the output of the Oracle about its average. Hence the whole Grover iteration can be formulated as

$$
\mathcal{G} \triangleq \mathcal{H P H O}=(2 \mathcal{H}|\mathbf{0}\rangle\langle\mathbf{0}| \mathcal{H}-\mathcal{H} \mathcal{I} \mathcal{H}) \mathcal{O},
$$

where $\mathcal{I}$ refers to the $N$-dimensional identity operation again, which together with the property of an unitary and Hermitian operator $\mathcal{H}=\mathcal{H}^{\dagger}=\mathcal{H}^{-1}$ simplifies $\mathcal{H} \mathcal{I} \mathcal{H}$ to $\mathcal{I}$. Utilizing expressions (4) and (5), equation (7) becomes

$$
\mathcal{G}=(2|\gamma\rangle\langle\gamma|-\mathcal{I}) \mathcal{O}=(2|\gamma\rangle\langle\gamma|-\mathcal{I})(\mathcal{I}-2|y\rangle\langle y|) .
$$

Generally, the second phase rotation operation is denoted by $\mathcal{U}_{\gamma}=(2|\gamma\rangle\langle\gamma|-\mathcal{I})$, where $|\gamma\rangle=\left[\begin{array}{llll}1 / \sqrt{N} & 1 / \sqrt{N} & \cdots & 1 / \sqrt{N}\end{array}\right]^{T}$ form (4), hence 


$$
\begin{aligned}
\mathbf{U}_{\gamma}=2\left[\begin{array}{c}
\frac{1}{\sqrt{N}} \\
\frac{1}{\sqrt{N}} \\
\vdots \\
\frac{1}{\sqrt{N}}
\end{array}\right]\left[\begin{array}{llll}
\frac{1}{\sqrt{N}} & \frac{1}{\sqrt{N}} & \cdots & \frac{1}{\sqrt{N}}
\end{array}\right]-\mathcal{I} \\
=\left[\begin{array}{cccc}
\frac{2}{N}-1 & \frac{2}{N} & \cdots & \frac{2}{N} \\
\frac{2}{N} & \frac{2}{N}-1 & \cdots & \frac{2}{N} \\
\vdots & & \ddots & \vdots \\
\frac{2}{N} & \cdots & \frac{2}{N} & \frac{2}{N}-1
\end{array}\right]
\end{aligned}
$$

One may apply the operation $\mathcal{U}_{\gamma}$ on a vector $|a\rangle=\left[\begin{array}{llll}a_{0} & a_{1} & \cdots & a_{N-1}\end{array}\right]^{T}$ and observe the amplitude of the $k^{t h}$ basis state

$$
\begin{aligned}
\left\langle k\left|\mathcal{U}_{\gamma}\right| a\right\rangle & =a_{0} \frac{2}{N}+a_{1} \frac{2}{N}+\cdots+\left(a_{k} \frac{2}{N}-a_{k}\right)+\cdots+ \\
& +a_{N} \frac{2}{N-1}= \\
& =2 \frac{1}{N} \sum_{i=0}^{N-1} a_{i}-a_{k}=2 \bar{a}-a_{k},
\end{aligned}
$$

which denotes a subtraction of the $y^{\text {th }}$ amplitude from the two times of the average $\bar{a}$ as it is depicted in Figure 5. The same observation is applied for every other $l=0, \ldots, N-1, l \neq y$ components. In the figure $y$ denotes the marked component $\left.f(x)\right|_{x=y}=1$, and $l$ the undesired ones, where $\left.f(x)\right|_{x=l}=0$, respectively. It is noticeable, as a result of the inversion on the average $\bar{a}$, the probability amplitude belonging to wanted index $y$ increases, whilst the amplitudes of other indexes fall off.

Invoking $\mathcal{G}$ several times, the algorithm amplifies the amplitude of the marked state and suppresses those for unmarked ones. If the probability amplitude of the marked state takes the value 1 , equivalently the values of others vanish, because $\sum_{i}\left|a_{i}\right|^{2}=1$. This process is called in the quantum world "Amplitude Amplification". However, the basic Grover algorithm can not guarantee that we can reach exactly $a_{y}=1$.

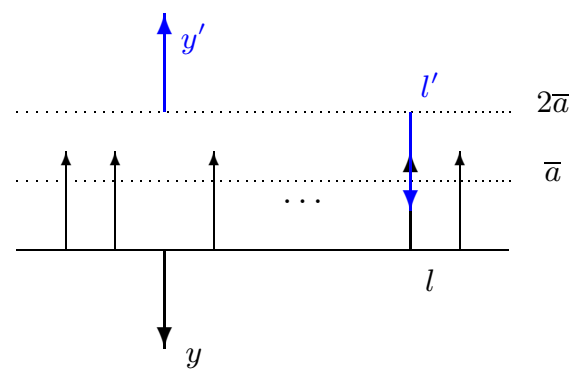

FiguRE 5. Inversion of the amplitude of a marked index $y$ and the unmarked one $l$ on the average $\bar{a}$ 


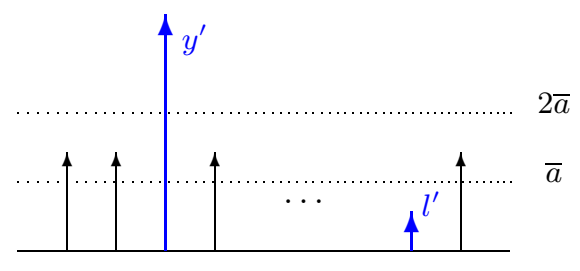

Figure 6. Probability amplitudes of a marked component $y$ and an unmarked one $l$ after the Oracle operation

2.2. Required Number of Iterations Multiple Marked States. As we discussed earlier amplitude amplification in Grover algorithm can not provide a sure success measurement of the marked index (i.e. $a_{y}=1$ ), because $a_{y}$ does not converge to 1 , instead it is a periodical function of the iteration steps. For that reason one should be able the predict the required number of iterations, which signifies: How many times should the Grover operator $(\mathcal{G})$ be applied to get $a_{y}$ as close as possible to 1 ? For this purpose we introduce a special geometrical description, by means we could also exploit an answer for databases with multiple marked entries $(M>1)$.

Let us form two basis state vectors from the marked and unmarked states as

$$
\begin{aligned}
|\alpha\rangle & \triangleq \frac{1}{\sqrt{N-M}} \sum_{x \in \bar{S}}|x\rangle, \\
|\beta\rangle & \triangleq \frac{1}{\sqrt{M}} \sum_{x \in S}|x\rangle, \\
S & : \text { Set of marked indexes }(f(x)=1)
\end{aligned}
$$

where (11) sums up the $(N-M)$ states $|x\rangle$ that do not lead to a solution of the search problem and (12) does the same with $M$ indexes $|x\rangle$ which lead to it. Variables $M$ and $N$ refer to the number of marked entries and the total number of entries in the database, respectively.

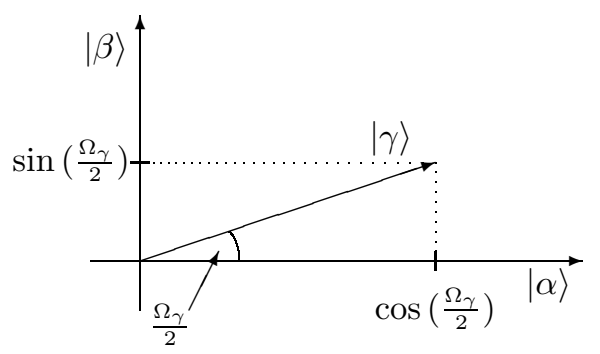

Figure 7. Projection of the initial state $|\gamma\rangle$ on the basis state vectors $|\alpha\rangle$ and $|\beta\rangle$ 
The initial state of the index register $|r\rangle$ before launching the search in (4) can be rewritten in the two dimensional space of the basis vectors as

$$
\begin{aligned}
|\gamma\rangle & =\frac{1}{\sqrt{N}} \sum_{x \in \bar{S}}|x\rangle+\frac{1}{\sqrt{N}} \sum_{x \in S}, \\
& =\sqrt{\frac{N-M}{N}}|\alpha\rangle+\sqrt{\frac{M}{N}}|\beta\rangle .
\end{aligned}
$$

The projections of $|\gamma\rangle$ onto the axes $|\alpha\rangle$ and $|\beta\rangle$ are given as

$$
\begin{gathered}
\cos \left(\frac{\Omega_{\gamma}}{2}\right)=\frac{\sqrt{\frac{N-M}{N}}}{1}, \\
\sin \left(\frac{\Omega_{\gamma}}{2}\right)=\frac{\sqrt{\frac{M}{N}}}{1},
\end{gathered}
$$

as shown in Figure 7. The denominators of (14) correspond to the fact that state $|\gamma\rangle$ has unit length.

As it was described in the previous subsection the basic Grover's database search algorithm consists of two transformations on the index register. The first $\mathcal{O}$ flips all the amplitudes of the marked states which corresponds to a reflection about axis $|\alpha\rangle$ because $|\beta\rangle$ contains only the indexes flipped by the Oracle.

The inversion about the average $(\mathcal{H P \mathcal { H }})$ transformation reflects its input state about $|\gamma\rangle$. Thus one Grover search $\left(\mathcal{G}^{1}\right)$ iteration agrees rotation of $|\gamma\rangle$ towards $|\beta\rangle$ with angle $\Omega_{\gamma}$. Within the frames of this geometrical interpretation our goal simplifies to rotating the index qregister as close to $|\beta\rangle$ as possible. This interpretation highlights the fact the fewer rotations than the optimal is as bad as more ones. The optimal number of iterations depends on the initial angle $\Omega_{\gamma} / 2$ between $|\gamma\rangle$ and $|\alpha\rangle$, as well as the number of the marked entries $M$ in the database.

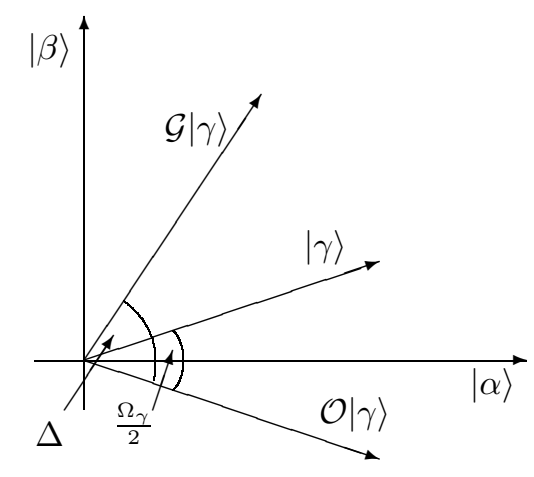

FiguRE 8. Geometrical interpretation of one Grover iteration

The rotation of initial state $|\gamma\rangle$ to the desired state $|\beta\rangle$ after $m$ evaluations of Grover operator is

$$
\mathcal{G}^{l}|\gamma\rangle=\cos \left(\frac{2 l+1}{2} \Omega_{\gamma}\right)|\alpha\rangle+\sin \left(\frac{2 l+1}{2} \Omega_{\gamma}\right)|\beta\rangle .
$$


It's worth performing a measurement if $\mathcal{G}^{l}|\gamma\rangle$ is equal to the state vector $|\beta\rangle$, i.e.

$$
\cos \left(\frac{2 l+1}{2} \Omega_{\gamma}\right)=0
$$

which can be transformed to

$$
\frac{2 l+1}{2} \cdot \Omega_{\gamma}=\frac{\pi}{2}+i \pi
$$

where $i=0,1, \ldots$. The optimal number of iterations is simply

$$
l_{\text {opt }_{i}}=\frac{\frac{\pi}{2}+i \pi-\frac{\Omega_{\gamma}}{2}}{\Omega_{\gamma}} .
$$

This result corresponds to the geometrical approach (Fig. 8) because the numerator represents the angle between the starting state $|\gamma\rangle$ and the final state $|\beta\rangle$ and the denominator substitutes the rotation step, respectively. Trivially we are forced to employ as few iterations as possible, therefore $\min _{i} l_{o p t_{i}}=l_{\text {opt }}$. Furthermore, in a quantum circuit we can apply only integer number of iterations $L_{o p t_{0}}$ calculated as

where

$$
L_{o p t_{0}}= \begin{cases}\left\lfloor l_{o p t_{0}}\right\rfloor & \text { if } d_{1} \leq d_{2} \\ \left\lfloor l_{o p t_{0}}\right\rfloor+1=\left\lceil l_{o p t_{0}}\right\rceil & \text { if } d_{1}>d_{2}\end{cases}
$$

$$
\begin{aligned}
& d_{1}=\left|\frac{\pi}{2}-\frac{2\left\lfloor l_{o p t_{0}}\right\rfloor+1}{2} \cdot \Omega_{\gamma}\right|, \\
& d_{2}=\left|\frac{\pi}{2}-\frac{2\left\lfloor l_{o p t_{0}}\right\rfloor+2}{2} \cdot \Omega_{\gamma}\right| .
\end{aligned}
$$

and $\lfloor x\rfloor$ denotes the closest integer to $x$ which is smaller or equal to $x$. It is easy to see that $d_{1}, d_{2} \leq \frac{\Omega_{\gamma}}{2}$. This definition of $L_{o p t_{0}}$ is originated from the geometrical representation and gives a clear explanation according to Fig. 9. From practical point of view a more compact form can be introduced:

$$
L_{o p t_{0}}=\operatorname{INT}\left(l_{o p t_{0}}\right) \text {, }
$$

where

$$
\operatorname{INT}(x)= \begin{cases}1 & \text { if } x>0.5 \\ 0 & \text { if } x \leq 0.5\end{cases}
$$

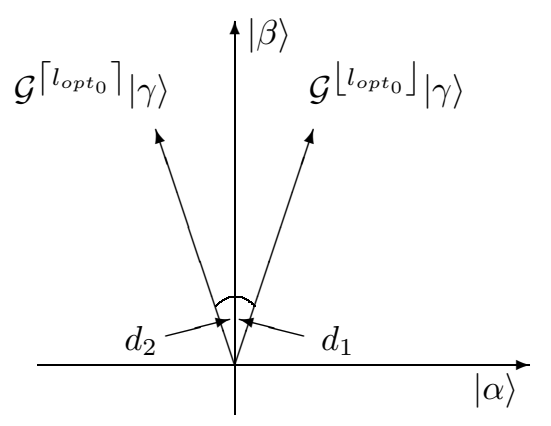

Figure 9. Visualization of the angles $d_{1}$ and $d_{2}$ 
In case of $M \ll N$

$$
\Omega_{\gamma} \simeq \sin \Omega_{\gamma}=2 \sqrt{\frac{M}{N}}
$$

yielding from (14), where the optimal number of evaluations is equal to

$$
L_{\text {opt }_{0}} \simeq \frac{\pi}{4} \sqrt{\frac{M}{N}}
$$

Now, we reached the surprising result $L_{o p t_{0}}=O\left(\sqrt{\frac{M}{N}}\right)$. From (16) with $i=0$ one can easily spot the initial angle $\Omega_{\gamma} / 2$ for a single query, which is $\Omega_{\gamma}=60^{\circ}$. This leads to a special relation of size of the database $N$ to the number of marked states $M$ (see Example 1.), which is

$$
N=4 M
$$

2.3. Probability of Error. Rotating $|\gamma\rangle L_{o p t_{0}}$ times with angle $\Omega_{\gamma}$, error may occur applying a measurement at the end of the database search, if $|r\rangle$ does not attain $|\beta\rangle$ exactly. It still remains a cosine scrap in (15), which is a projection of $\mathcal{G}^{l}|\gamma\rangle$ on $|\alpha\rangle$ describing the probability amplitude of the final state being in the unmarked basis vector state. Thus the probability of error resulting from basic Grover's search algorithm is calculated as

$$
P_{\varepsilon}=\cos ^{2}\left(\frac{\left(2 L_{\text {opt }_{0}}+1\right) \Omega_{\gamma}}{2}\right)
$$

and the Probability of Success

$$
P_{s}=1-P_{\varepsilon}=\sin ^{2}\left(\frac{\left(2 L_{o p t_{0}}+1\right) \Omega_{\gamma}}{2}\right) .
$$

Since $d_{1}, d_{2} \leq \frac{\Omega_{\gamma}}{2}$ therefore $P_{\varepsilon} \leq \sin ^{2}\left(\frac{\Omega_{\gamma}}{2}\right)$. Substituting $\Omega_{\gamma}$ according to (18), $P_{\varepsilon} \leq \frac{M}{N}$. Finally, we have an important remark. Equation (16) highlights the fact that we have a tradeoff between $P_{\varepsilon}$ and $L_{o p t_{i}}$, because $l_{o p t_{0}}$ is only a local minimum place of number of iterations. This inspire us to search for the global optimum in function of $i$ if the accuracy of the measurement is playing more important role than the number of Grover steps. Fortunately, the generalized Grover search algorithm is able to guarantee sure success measurement using about $L_{o p t_{0}}$ iterations. However, if we are restricted to buy the original Grover quantum search circuit at the grocer's it is worth seeking for the global optimum.

\section{Several Remarks on the Proportion of Marked States and the SIZE OF THE DATABASE}

In previous subsections it was introduced a proportion $\sqrt{M / N}$ between the number of marked states $M$ and the dimension of search space $N$, that makes a significant contribution to the effectiveness of the search algorithm. The initial state $|\gamma\rangle$ according to (14), the angle of rotation $\Omega_{\gamma}$ as well as the required number of iterations $l_{\text {opt }}$ from (16), which include the speed and the performance $P_{\varepsilon}$ of the search in (20), are all depending from this ratio. The larger it is the less is $\Omega_{\gamma}$ and larger is $l$ as depicted in Fig. 11 and 12. Consequently, finding a slight number of marked entries in a huge database, the initial state may converge to $|\beta\rangle$ with higher accuracy but at the cost of more iterations, keeping off the oscillation about 
it, resulting in a lower $P_{\varepsilon}$. However, one should keep in mind that the number of iterations is still far lower as in classical searching algorithm even in these situations. Let us examine several bounds for $\sqrt{M / N}$.

- It is unambiguous that $\sqrt{M / N}$ must be less or equal one, since it would stand for more marked states than the entire number of entries in the database.

- A trivial solution is when $\sqrt{M / N}$ is equal to one, which means an initial angle $\frac{\Omega_{\gamma}}{2}=\frac{\pi}{2}$, which implies no needed rotation. Each measurement carries out the proper solution, however, this case happens occasionally.

- Let us determine the values for $\frac{M}{N}$ when it is worth performing the measurement directly without any iteration, i.e. $L_{\mathrm{opt}_{0}}=0$. According to (16) and taking into account the property $\sin (x)=\cos \left(\frac{\pi}{2}-x\right)$ and (14),

$$
l_{o p t_{0}}=\frac{\frac{\pi}{2}-\frac{\Omega_{\gamma}}{2}}{2 \frac{\Omega_{\gamma}}{2}}=\frac{\arccos \left(\sqrt{\frac{M}{N}}\right)}{2 \arcsin \left(\sqrt{\frac{M}{N}}\right)}<0.5,
$$

or

$$
\arccos \left(\sqrt{\frac{M}{N}}\right)<\arcsin \left(\sqrt{\frac{M}{N}}\right) .
$$

Employing simple trigonometrical calculus (see Fig. 10) it turns out a solution

$$
\frac{\sqrt{2}}{2}<\sqrt{\frac{M}{N}} \leq 1,
$$

from which the bounds become

$$
\frac{1}{2}<\frac{M}{N} \leq 1
$$

Since, $\sin \left(\frac{\Omega_{\gamma}}{2}\right)=\frac{M}{N}$, therefore expression (22) is equivalent to $\frac{\pi}{4}<\Omega_{\gamma} \leq \frac{\pi}{2}$ in terms of initial state angle. If $L_{o p t_{0}}=0$ we have two choices. Either we perform measurement without any iteration. The probability of success is in this case $P_{s}=\frac{M}{N} \geq \frac{1}{2}$. Or we double the size of the database using unmarked entries. Hence, $\frac{M}{N} \Rightarrow \frac{M}{2 N}<\frac{1}{2}$ and we can increase the accuracy $\left(P_{s}\right)$ at the cost of more iteration steps. Clearly speaking the size of the database does not need to be increased instead only one qubit should be added to the index qregister and the Oracle should be programmed in that way it answers $f(x)=0$ for $x>N$ !

As an example let $M$ be chosen to $0.75 \cdot 2^{32}$, whereas the size of the database should be 32 qubits long. The proportion $\frac{M}{N}=0.75$. Following equations (16) and (20), there is no need for anz rotation and at the same time $P_{\varepsilon}=0.25$. Adding a single qubit to the index register $N$ becomes $2^{33}$, whereby $\frac{M}{N}=0.075$. For a proper solution one should apply the Grover's search iteration twice to obtain an error probability $P_{\varepsilon}=0.033$, which is $\sim 86.6 \%$ better than previously at a cost of only for 2 iterations. Finally observing Fig. 11 and 12 one can draw the conclusion that in typical scenarios where $M \ll N$ the probability of error is dramatically reduced. 


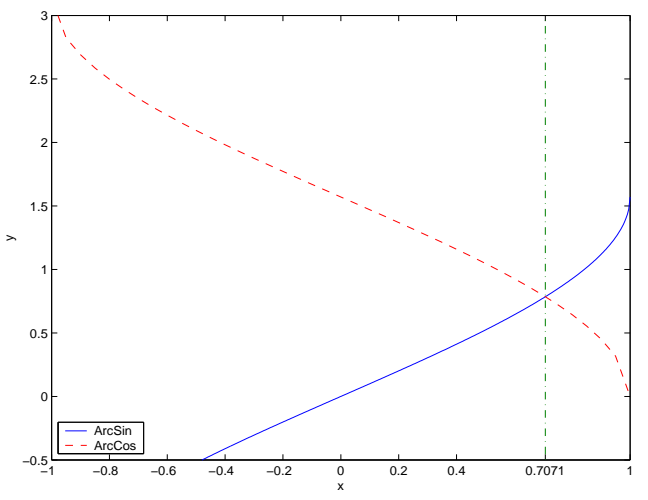

FiguRE 10. Intersection of $\arccos (x)$ and $\arcsin (x)$
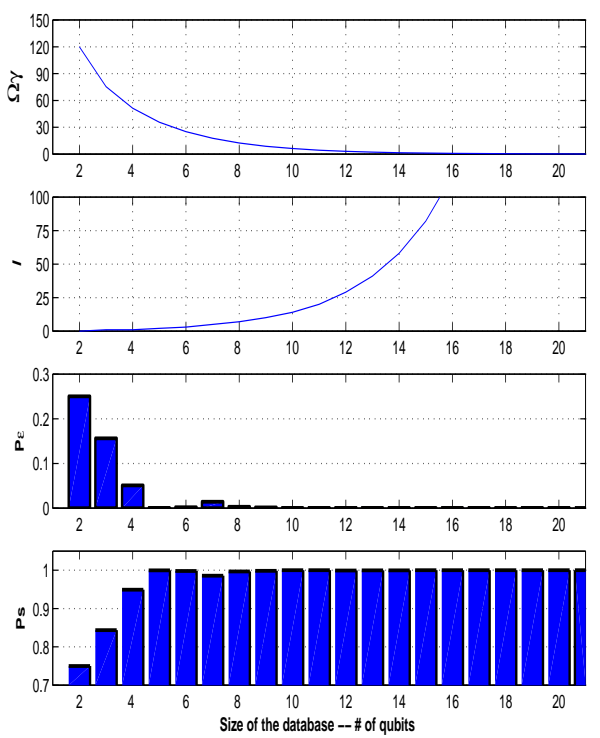

FiguRE 11. Varying of the rotation angle $\Omega_{\gamma}$, the number of iterations $l$, the Probability of Error $P_{\varepsilon}$ and the Probability of Success $\left(P_{s}\right)$ with increasing size of the database. $M=3, N=2^{(2, \ldots, 19)}$

\section{Generalized Grover Algorithm}

During the analysis of the basic Grover algorithm we found that one should keep an eye on a contradictory fact, that the result of a search should be obtained using as less iterations as possible meanwhile achieving high accuracy. For that reason a generalized Grover's search algorithm is introduced and discussed in the next subsections.

4.1. Motivation. According to the utilization of Grover's database search algorithm in practice, larger quantum systems should be taken into account where the input index register of the algorithm is given as an arbitrary output state of a 

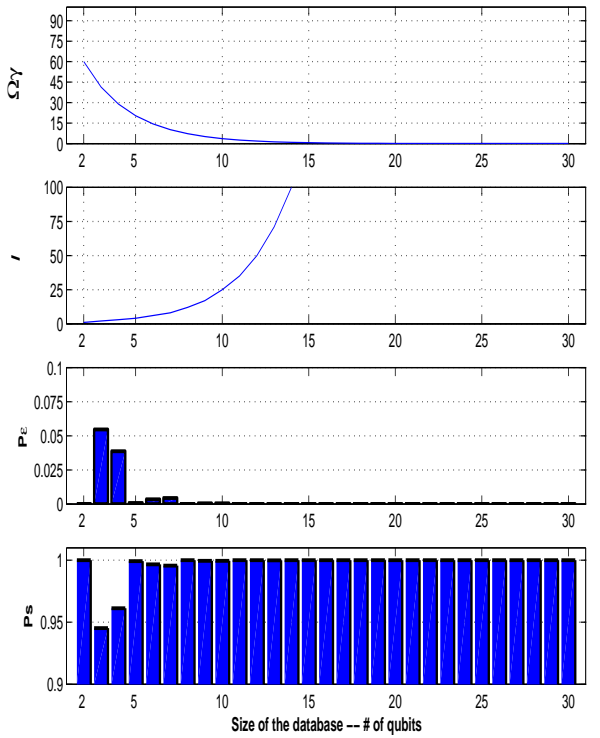

FiguRE 12. Varying of the rotation angle $\Omega_{\gamma}$, the number of iterations $l$, the Probability of Error $P_{\varepsilon}$ and the Probability of Success $\left(P_{s}\right)$ with increasing size of the database. $M=1, N=2^{(2, \ldots, 30)}$

former circuit and the output of the algorithm can feed another circuit without any measurement. Hence the exact knowledge of the index register after the final iteration is of great significance.

4.2. Generalization of the Original Grover's Database Search Algorithm. In order to give the generalization of the basic Grover algorithm we rewrite in this subsection the original relations and introduce some new definitions, respectively.

In Section 2.1 the Grover operator was originally defined as

$$
\mathcal{G} \triangleq \mathcal{H P H O}
$$

where

$$
\begin{gathered}
\mathcal{P} \triangleq(2|\mathbf{0}\rangle\langle\mathbf{0}|-\mathcal{I}), \\
\mathcal{O} \triangleq \mathcal{I}-2|y\rangle\langle y|
\end{gathered}
$$

and

$$
|\gamma\rangle \triangleq \mathcal{H}^{\otimes^{n}}|\mathbf{0}\rangle_{n}=\frac{1}{\sqrt{N}} \sum_{x=0}^{N-1}|x\rangle,
$$

are determined as defined in (4), (5), where $N=2^{n}$ denotes the number of items in the database, again.

Henceforth let us apply some new necessary definitions and practical considerations.

(1) From now onward $\mathcal{H}$ should be replaced by an arbitrary unitary transformation $\mathcal{U}(\mathcal{H} \rightarrow \mathcal{U})$. 
(2) The Oracle $(\mathcal{O})$ should rotate the probability amplitude of the marked items in the index register with an angle of $\phi$ in lieu of $\pi$, set originally, where $\phi \in[-\pi, \pi]$. Thus (5) is altered to

$$
\mathcal{O} \rightarrow \mathcal{I}_{\beta} \triangleq \mathcal{I}+\left(e^{\jmath \phi}-1\right) \sum_{x \in S}|y\rangle\langle y| .
$$

(3) Analogue to the Oracle above, the controlled phase gate $(\mathcal{P})$ which was originally based on the state $|\mathbf{0}\rangle$ have to be founded on an arbitrary basis state $|\eta\rangle$ resulting in a multiplication by $e^{\jmath \theta}$ instead of -1 , where $\theta \in[-\pi, \pi]$. In more exact mathematical formalism

$$
\mathcal{P} \rightarrow \mathcal{I}_{\eta} \triangleq \mathcal{I}+\left(e^{\jmath \theta}-1\right)|\eta\rangle\langle\eta|
$$

(4) Furthermore the initial state of index register is considered as

$$
|\gamma\rangle \triangleq \sum_{x=0}^{2^{n}-1} \gamma_{x}|x\rangle,
$$

where $\sum_{x=0}^{\left(2^{n}-1\right)}\left|\gamma_{x}\right|^{2}=1$ as appropriate.

(5) Finally the two basis vectors $|\alpha\rangle$ and $|\beta\rangle$ consisting of the indexes leading to unmarked solutions and of the indexes ending in a marked entry should be redefined, that were originally set in (11) and (12), respectively

$$
\begin{aligned}
|\alpha\rangle & =\frac{1}{\sqrt{\sum_{x \in \bar{S}}\left|\gamma_{x}\right|^{2}}} \sum_{x \in \bar{S}} \gamma_{x}|x\rangle, \\
|\beta\rangle & =\frac{1}{\sqrt{\sum_{x \in S}\left|\gamma_{x}\right|^{2}}} \sum_{x \in S} \gamma_{x}|x\rangle,
\end{aligned}
$$

where $\bar{S}$ represents the complementary set of indexes to $S$.

4.2.1. Properties of the Newly Defined Basis Vectors. We digress for a moment to discuss the properties of newly defined basis vectors $|\alpha\rangle$ and $|\beta\rangle$.

- Vector $|\alpha\rangle$ is built from items $x$ which do not lead to a solution and therefore there do not lie in the set $S,|\alpha\rangle=\sum_{x \in \bar{S}} \alpha_{x}|x\rangle$.

- Observing the new basis vectors $|\alpha\rangle$ and $|\beta\rangle$ the orthogonality is still given between them, $\langle\alpha \mid \beta\rangle=0$, since during the pairwise multiplication within the inner product one of the factors is always zero.

- It is obvious that neither $|\alpha\rangle$ nor $|\beta\rangle$ could be $|\mathbf{0}\rangle$, except the extreme and unambiguous cases, when the search can not lead to a solution (since $|\beta\rangle=0)$ and the case $(|\alpha\rangle=0)$ where a search would be senseless since a measurement shows the result with a probability of one, immediately.

Regarding the definitions in $(23)$ and $(23)$ the generalized Grover operation $(\mathcal{G} \rightarrow \mathcal{Q})$ looks like as follows

$$
\begin{aligned}
\mathcal{Q} & =-\mathcal{U} \mathcal{I}_{\eta} \mathcal{U}^{\dagger} \mathcal{I}_{\beta}=-\mathcal{U}\left(\mathcal{I}+\left(e^{\jmath \theta}-1\right)|\eta\rangle\langle\eta|\right) \mathcal{U}^{\dagger} \mathcal{I}_{\beta} \\
& =-\left(\mathcal{U} \mathcal{I U}^{-1}+\left(e^{\jmath \theta}-1\right) \mathcal{U}|\eta\rangle\langle\eta| \mathcal{U}^{\dagger}\right) \mathcal{I}_{\beta} \\
\mathcal{Q} & =-\left(\mathcal{I}+\left(e^{\jmath \theta}-1\right)|\mu\rangle\langle\mu|\right) \mathcal{I}_{\beta},
\end{aligned}
$$

where

$$
|\mu\rangle \triangleq \mathcal{U}|\eta\rangle
$$


and relation $\mathcal{U}^{\dagger}=\mathcal{U}^{-1}$ is exploited in consequence of the unitary operation property, respectively.

Before continuing our examinations, let us prove the completeness of the search.

Lemma 4.1. If the state vectors $|\alpha\rangle$ and $|\beta\rangle$ are defined according to (26) and (27), as well as the unitary operator $\mathcal{U}$ and an arbitrary state $|\eta\rangle$ are taken in such a way that $\mathcal{U}|\eta\rangle$ lies within the vector space spanned by the state vectors $|\alpha\rangle$ and $|\beta\rangle$, then the generalized Grover operator $(\mathcal{Q})$ preserves this 2-dimensional vector space. For any $|v\rangle \in V, \mathcal{Q}|v\rangle \in V$ is true.

Proof. Following the geometrical definition of inner product, the length of the projection of $\mathcal{U}|\eta\rangle$ on vector $|\beta\rangle$ is $\langle\beta|\mathcal{U}| \eta\rangle \cdot|\beta\rangle$. Since $\mathcal{U}|\eta\rangle$ is defined in the vector space $V$, the vector $\mathcal{U}|\eta\rangle-\langle\beta|\mathcal{U}| \eta\rangle|\beta\rangle$ is parallel to $|\alpha\rangle$ and it is exactly the ,length of the vector" times $|\alpha\rangle$,

$$
\mathcal{U}|\eta\rangle-\langle\beta|\mathcal{U}| \eta\rangle|\beta\rangle=\sqrt{1-|\langle\beta|\mathcal{U}| \eta\rangle| \beta\rangle\left.\right|^{2}}|\alpha\rangle
$$

Hence, $|\alpha\rangle$ can be expressed as

$$
|\alpha\rangle=\frac{1}{\sqrt{1-|\langle\beta|\mathcal{U}| \eta\rangle|^{2}}}(\mathcal{U}|\eta\rangle-\langle\beta|\mathcal{U}| \eta\rangle|\beta\rangle) .
$$

Vector $|\mu\rangle$ is considered as an arbitrary unit vector in $V$

$$
|\mu\rangle_{2}=\cos (\Omega)|\alpha\rangle+\sin (\Omega) e^{\jmath \Lambda}|\beta\rangle
$$

where $\Omega, \Lambda \in[-\pi, \pi]$.

As the next step the generalized Grover operator should be given in $V$.

$$
\mathcal{Q}|\beta\rangle=-\mathcal{U} \mathcal{I}_{\eta} \mathcal{U}^{\dagger} \mathcal{I}_{\beta}|\beta\rangle,
$$

the required two dimensional Grover matrix and the basis vectors are searched in the form of

$$
\mathbf{Q}_{\mathbf{2}}=\left[\begin{array}{ll}
Q_{11} & Q_{12} \\
Q_{21} & Q_{22}
\end{array}\right] .
$$

As $\mathcal{I}_{\beta}$ multiplies every indexes leading to a marked entry by $e^{\jmath \phi}$,

$$
\mathcal{I}_{\beta}|\beta\rangle=e^{\jmath \phi}|\beta\rangle \text {. }
$$

Equation (31) alters considering (32) to

$$
\begin{aligned}
\mathcal{Q}|\beta\rangle & =-\left[\mathcal{U} \mathcal{I U}^{-1}+\left(e^{\jmath \theta}-1\right)|\mu\rangle\langle\mu|\right] e^{\jmath \phi}|\beta\rangle \\
& =-e^{\jmath \phi}|\beta\rangle\left(e^{\jmath \theta}-1\right) e^{\jmath \phi} \mathcal{U}|\eta\rangle\left\langle\eta\left|\mathcal{U}^{-1}\right| \beta\right\rangle \\
& =-e^{\jmath \phi}\left(\left(e^{\jmath \theta}-1\right)\langle\mu \mid \beta\rangle|\mu\rangle+|\beta\rangle\right) .
\end{aligned}
$$

Applying (30) and the relation $\langle\mu \mid \beta\rangle=\langle\beta \mid \mu\rangle^{*}=\sin (\Omega) e^{-\jmath \Lambda}$, where $|\mu\rangle$ is according to $(29)$,

$$
\begin{aligned}
\mathcal{Q}|\beta\rangle= & -e^{\jmath \phi}\left(e^{\jmath \theta}-1\right) \sin (\Omega) e^{-\jmath \Lambda} \\
= & \underbrace{\left(\cos (\Omega)|\alpha\rangle+\sin (\Omega) e^{\jmath \Lambda}|\beta\rangle\right)-e^{\jmath \phi}|\beta\rangle}_{Q_{21}} \\
& +\underbrace{-e^{\jmath \phi}\left(e^{\jmath \theta}-1\right) \sin (\Omega) \cos (\Omega) e^{-\jmath \Lambda}}_{Q_{22}}|\alpha\rangle
\end{aligned}
$$


Moreover, the further two entries in $\mathbf{Q}$

$$
\mathcal{Q}|\alpha\rangle=-\left[\mathcal{I}+\left(e^{\jmath \theta}-1\right)|\mu\rangle\left\langle\mu\left|\mathcal{I}_{\beta}\right| \alpha\right\rangle\right]
$$

where $\mathcal{I}_{\beta}|\alpha\rangle=|\alpha\rangle$, because only the indexes leading to a solution are rotated by $\mathcal{I}_{\beta}$ otherwise will be maintained, which ends to

$$
\langle\mu \mid \alpha\rangle=\langle\alpha \mid \mu\rangle^{*}=\cos (\Omega) .
$$

Thus, similar to $(34)$

$$
\begin{aligned}
\mathcal{Q}|\alpha\rangle & =\underbrace{-\left[1+\left(e^{\jmath \theta}-1\right) \cos ^{2}(\Omega)\right]}_{Q_{11}}|\alpha\rangle \\
& +\underbrace{-\left[\left(e^{\jmath \theta}-1\right) \cos (\Omega) \sin (\Omega) e^{\jmath \Lambda}\right]}_{Q_{12}}|\beta\rangle
\end{aligned}
$$

Now, from (34) and (37) we conclude that $\mathcal{Q}|\alpha\rangle$ and $\mathcal{Q}|\beta\rangle$ is remained in the vector space $V$, therefore all the linear superposition

$$
|v\rangle=a|\alpha\rangle+b|\beta\rangle
$$

of $|\alpha\rangle$ and $|\beta\rangle$ transformed by $\mathcal{Q}$ still remained in the vector space $V$.

Based on equations (34) and (37) we have matrix $\mathbf{Q}_{\mathbf{2}}$ in a suitable two dimensional form

$$
\begin{array}{r}
\mathbf{Q}_{\mathbf{2}}=-\left[\begin{array}{cc}
1+\left(e^{\jmath \theta}-1\right) \cos ^{2}(\Omega) & e^{\jmath \phi}\left(e^{\jmath \theta}-1\right) \sin (\Omega) \cos (\Omega) e^{-\jmath \Lambda} \\
\left(e^{\jmath \theta}-1\right) \cos (\Omega) \sin (\Omega) e^{\jmath \Lambda} & e^{\jmath \phi}\left[1+\left(e^{\jmath \theta}-1\right) \sin ^{2}(\Omega)\right]
\end{array}\right] \\
=-\left[\begin{array}{cc}
e^{\jmath \theta} \cos ^{2}(\Omega)+\sin ^{2}(\Omega) & e^{\jmath \phi} e^{-\jmath \Lambda}\left(e^{\jmath \theta}-1\right) \frac{\sin (2 \Omega)}{2} \\
\left(e^{\jmath \theta}-1\right) e^{\jmath \Lambda} \frac{\sin (2 \Omega)}{2} & e^{\jmath \phi}\left[e^{\jmath \theta} \sin ^{2}(\Omega)+\cos ^{2}(\Omega)\right]
\end{array}\right] .
\end{array}
$$

From this point forward $\mathbf{Q}$ always refers to the two dimensional Grover matrix, if otherwise not indicated.

\subsection{Required Number of Iterations in the Generalized Grover's Search} Algorithm. After being acquainted with the 2-dimensional generalized Grover operator $\mathcal{Q}$, the optimal number of iterations $l_{\text {opt }}$ during a search should be derived. Thus, we follow the assumption

$$
\left\langle\alpha\left|\mathcal{Q}^{l_{o p t}}\right| \gamma\right\rangle=0
$$

which stands for having an index register orthogonal to the vector including all the indexes which do not lead to a solution. Because $|\alpha\rangle$ and $|\beta\rangle$ are orthogonal, this assumption can be interpreted as $\mathcal{Q}^{l_{\text {opt }}}|\gamma\rangle$ is parallel to $|\beta\rangle$ i.e. $\mathcal{Q}^{l_{\text {opt }}}|\gamma\rangle=e^{\jmath \delta}|\beta\rangle$. In this case sure success can be reached after a measurement. Since $\mathcal{Q}$ is an unitary operator and therefore it is a normal operator, hence it has a spectral decomposition

$$
\mathcal{Q}=q_{1}\left|\psi_{1}\right\rangle+q_{2}\left|\psi_{2}\right\rangle
$$

where $q_{1,2}$ denote the eigenvalues of $\mathcal{Q}$ and $\left|\psi_{1,2}\right\rangle$ stand for the eigenvectors of $\mathcal{Q}$, respectively, with the concatenation

$$
\mathcal{Q}\left|\psi_{1,2}\right\rangle=q_{1,2}\left|\psi_{1,2}\right\rangle
$$


where $\left\langle\psi_{1} \mid \psi_{2}\right\rangle=0$, because of the orthogonality between the eigenvectors. The eigenvalues determined from the characteristic equation $\operatorname{det}\{\mathbf{Q}-q \mathbf{I}\}=0$ (see Appendix A) are

$$
q_{1,2}=-e^{\jmath\left(\frac{\theta+\phi}{2} \pm \Delta\right)} .
$$

In addition we claim the following restriction on the angle $\Delta$

$$
\cos \Delta=\cos \left(\frac{\theta-\phi}{2}\right)+\sin ^{2}(\Omega)\left(\cos \left(\frac{\theta+\phi}{2}\right)-\cos \left(\frac{\theta-\phi}{2}\right)\right) .
$$

In possession of the eigenvalues the next step towards the optimal number of iterations is the determination of the normalized eigenvectors $\left|\psi_{1,2}\right\rangle$, which are

$$
\begin{array}{r}
\left|\psi_{1}\right\rangle=\cos (z) e^{\jmath\left(\frac{\phi}{2}-\Lambda\right)}|\alpha\rangle+\sin (z)|\beta\rangle, \\
\left|\psi_{2}\right\rangle=-\sin (z) e^{\jmath\left(\frac{\phi}{2}-\Lambda\right)}|\alpha\rangle+\cos (z)|\beta\rangle,
\end{array}
$$

where

$$
\sin ^{2}(z)=\frac{\sin ^{2}(2 \Omega) \sin ^{2}\left(\frac{\theta}{2}\right)}{2\left(1-\cos \left(\frac{\theta}{2}\right) \cos \left(\frac{\phi}{2}-\Delta\right)-2 \cos (2 \Omega) \sin \left(\frac{\theta}{2}\right) \sin \left(\frac{\phi}{2}-\Delta\right)\right)} .
$$

The detailed derivation of the eigenvectors and eigenvalues can be found in the Appendix (A and B).

Due to the spectral decomposition and the relation $\left\langle\psi_{1} \mid \psi_{2}\right\rangle=\left\langle\psi_{2} \mid \psi_{1}\right\rangle=0$,

$$
\begin{aligned}
\mathcal{Q}^{l} & =q_{1}^{l}\left|\psi_{1}\right\rangle\left\langle\psi_{1}\left|+q_{2}^{l}\right| \psi_{2}\right\rangle\left\langle\psi_{2}\right| \\
& =(-1)^{l} e^{\jmath l\left(\frac{\theta+\phi}{2}\right)} . \\
& \cdot\left[\begin{array}{cc}
e^{\jmath\left(\frac{\phi}{2}-\Lambda\right)}\left(e^{\jmath l \Delta} \cos ^{2}(z)+e^{-\jmath l \Delta} \sin ^{2}(z)\right) & \jmath \sin (l \Delta) \sin (2 z) e^{\jmath\left(\frac{\phi}{2}-\Lambda\right)} \\
\jmath \sin (l \Delta) \sin (2 z) e^{-\jmath\left(\frac{\phi}{2}-\Lambda\right)} & e^{\jmath l \Delta} \sin ^{2}(z)+e^{-\jmath l \Delta} \cos ^{2}(z)
\end{array}\right] .
\end{aligned}
$$

Using (45), $l$ can be derived by

$$
\left\langle\alpha\left|\mathcal{Q}^{l}\right| \gamma\right\rangle=0,
$$

which is fulfilled if both - the real and the imaginary- part of (46) are equal to zero. Let $|\gamma\rangle$ be defined as an arbitrary unit vector in $V$

$$
|\gamma\rangle=\cos \left(\Omega_{\gamma}\right)|\alpha\rangle+\sin \left(\Omega_{\gamma}\right) e^{\jmath \Lambda_{\gamma}}|\beta\rangle .
$$

Thus,

$$
\begin{aligned}
\left\langle\alpha\left|\mathcal{Q}^{l}\right| \gamma\right\rangle & =\cos \left(\Omega_{\gamma}\right) Q_{11}^{l}+\sin \left(\Omega_{\gamma}\right) e^{\jmath \Lambda_{\gamma}} Q_{12}^{l}= \\
& =\cos \left(\Omega_{\gamma}\right)\left[e^{\jmath l \Delta} \cos ^{2}(z)+e^{-\jmath l \Delta} \sin ^{2}(z)\right]+ \\
& +\jmath e^{\jmath\left(\frac{\phi}{2}-\Lambda+\Lambda_{\gamma}\right)} \sin (l \Delta) \sin (2 z) \sin \left(\Omega_{\gamma}\right)=0
\end{aligned}
$$

The real part of (47) is

$$
\begin{aligned}
\Re\left\{\left\langle\alpha\left|\mathcal{Q}^{l}\right| \gamma\right\rangle\right\}= & \cos \left(\Omega_{\gamma}\right) \underbrace{\left[\cos (l \Delta) \cos ^{2}(z)+\cos (l \Delta) \sin ^{2}(z)\right]}_{\cos (l \Delta)}- \\
& -\sin \left(\Lambda_{\gamma}-\Lambda+\frac{\phi}{2}\right) \sin (l \Delta) \sin (2 z) \sin \left(\Omega_{\gamma}\right)=
\end{aligned}
$$




$$
=\cos \left(\Omega_{\gamma}\right) \cos (l \Delta)-\sin \left(\Omega_{\gamma}\right) \sin (l \Delta) \sin (2 z) \sin \left(\Lambda_{\gamma}-\Lambda+\frac{\phi}{2}\right)=0
$$

whereas the imaginary one is equal to

$$
\begin{aligned}
\Im\left\{\left\langle\alpha\left|\mathcal{Q}^{l}\right| \gamma\right\rangle\right\}=\cos \left(\Omega_{\gamma}\right) & \underbrace{\left[\sin (l \Delta) \cos ^{2}(z)-\sin (l \Delta) \sin ^{2}(z)\right]}_{\sin (l \Delta) \cos (2 z)}+ \\
& +\cos \left(\Lambda_{\gamma}-\Lambda+\frac{\phi}{2}\right) \sin (l \Delta) \sin (2 z)=0 .
\end{aligned}
$$

Since, $\sin (l \Delta) \neq 0$, the imaginary part becomes

$$
=\cos \left(\Lambda_{\gamma}-\Lambda+\frac{\phi}{2}\right) \sin (2 z) \sin \left(\Omega_{\gamma}\right)+\cos \left(\Omega_{\gamma}\right) \cos (2 z)=0
$$

4.3.1. Matching Condition. Equation (49) does not depend on $l$, which makes it appropriate to determine the so called ,, matching condition" (MC), the relationship between $\theta$ and $\phi$,

$$
\cos \left(\Lambda_{\gamma}-\Lambda+\frac{\phi}{2}\right)=-\cot (2 z) \cot \left(\Omega_{\gamma}\right)
$$

and thus

$$
\tan \left(\frac{\phi}{2}\right)=\frac{\cos (2 \Omega)+\sin (2 \Omega) \cdot \tan \left(\Omega_{\gamma}\right) \cos \left(\Lambda-\Lambda_{\gamma}\right)}{\cot \frac{\phi}{2}-\tan \left(\Omega_{\gamma}\right) \sin (2 \Omega) \sin \left(\Lambda-\Lambda_{\gamma}\right)} .
$$

It is worth emphasizing, that according to (42) $\Delta$ is $4 \pi$ periodical in function of $\theta$, which includes clasping a $4 \pi$ range of $\phi$ by determining $\phi$ form $\theta$, since $\Delta$ is also depends on $\phi$. This seems to be in contradiction that the eigenvalues $q_{1,2}$ should be $2 \pi$ periodical in $\theta$ and $\phi$. This problem can be eliminated if $\phi$ is calculated for the range $[-2 \pi, 2 \pi]$ in function of $\theta \in[-2 \pi, 2 \pi]$. Practically $\pm 2 \pi$ should be added to $\phi$ if it has a cut-off in the function.

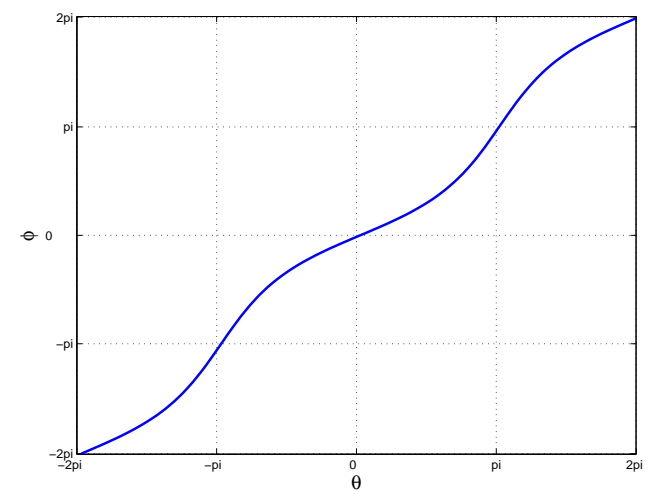

Figure 13. The angles $\phi$ vs. $\theta$ without and with correction

The points where $\phi(\theta)$ has cut-off's within the range of $[-\pi, \pi]$ are the points where

$$
\phi= \pm \pi \Rightarrow \tan \left(\frac{\phi}{2}\right)= \pm \infty \text {. }
$$


Inasmuch as, the numerator of the matching condition in (50) is constant in $\theta$, the denominator have to be zero to achieve the condition $\phi= \pm \infty$. The cut-off angles $\theta_{\mathrm{co}_{1,2}}$ can be derived from denominator of (50) as follows. From (50),

$$
\cot \left(\frac{\phi}{2}\right)=\tan \left(\Omega_{\gamma}\right) \sin (2 \Omega) \sin \left(\Lambda-\Lambda_{\gamma}\right)
$$

the cut-off angles in $[-2 \pi, 2 \pi]$ are

$$
\begin{aligned}
& \theta_{c o_{1}}=2 \arctan \left(\tan \left(\Omega_{\gamma}\right) \sin (2 \Omega) \sin \left(\Lambda-\Lambda_{\gamma}\right)\right), \\
& \theta_{c o_{2}}=\theta_{c o_{1}} \pm 2 \pi .
\end{aligned}
$$

By means of this correction $2 \pi$ periodicity of $\Delta$ is achieved, hence, the eigenvalues and eigenvectors of $\mathcal{Q}$, even $\mathcal{Q}$ itself can boast a $2 \pi$ periodicity in $\theta$.

4.3.2. Optimal Number of Iterations. Now, the way is open to determine $l$ from (49) which provides a measurement with $P_{\varepsilon}=0$. The matching condition (50) should be also considered leading to

$$
\cos \left(l \Delta+\arcsin \left(\sin \left(\frac{\phi}{2}-\Lambda+\Lambda_{\gamma}\right) \sin \left(\Omega_{\gamma}\right)\right)\right)=0
$$

which is equivalent to

$$
l \Delta= \pm \frac{\pi}{2} \pm i \pi-\arcsin \left(\sin \left(\frac{\phi}{2}-\Lambda+\Lambda_{\gamma}\right) \sin \left(\Omega_{\gamma}\right)\right)
$$

where $\pm i \pi$ is omitted from the right hand side, because it would result in a bigger $l$. Unlike the basic algorithm where $i>0$ could result in a more accurate measurement in case of the generalized algorithm $i=0$ provides $P_{\varepsilon}=0$, Expression (53) can be interpreted in the following way. The generalized Grover operator $(\mathcal{Q})$ rotates the new initial state $|\gamma\rangle^{\prime}$ with the initial angle $\Omega_{\gamma}=\arcsin \left(\sin \left(\frac{\phi}{2}-\Lambda+\Lambda_{\gamma}\right) \sin \left(\Omega_{\gamma}\right)\right)$ in a plane $V^{\prime}$ spanned by the basis vectors $|\alpha\rangle^{\prime}$ and $|\beta\rangle^{\prime}$ with a single rotation angle $\Delta$ towards $|\beta\rangle^{\prime}$ as it is depicted in Fig. 14. It shall be remarked that $|\alpha\rangle^{\prime}$ and $|\beta\rangle^{\prime}$ are real valued axes while $|\alpha\rangle$ and $|\beta\rangle$ are complex valued.

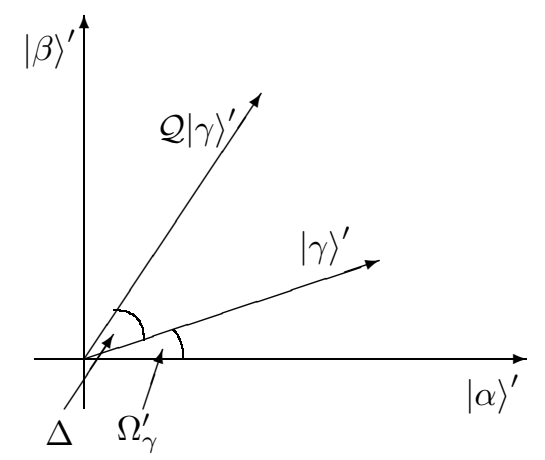

FIGURE 14. Geometrical interpretation of the generalized Grover iteration 
Because of the arbitrary sing of $\sin \left(\frac{\phi}{2}-\Lambda+\Lambda_{\gamma}\right), \Omega_{\gamma}^{\prime}$ can take different values, depending on

$$
\nu=\arcsin \left(\sin \left(\frac{\phi}{2}-\Lambda+\Lambda_{\gamma}\right) \sin \left(\Omega_{\gamma}\right)\right),
$$

where $\arcsin (\cdot)$ and $\arccos (\cdot)$ are defined as

$$
|\arcsin (\cdot)| \leq \frac{\pi}{2}
$$

and

$$
0 \leq \arccos (\cdot) \leq \pi
$$

If $\nu$ is positive the initial angle $\Omega_{\gamma}^{\prime}$ could be $(\pi-\nu)$ or $(\nu)$, in the other case the possible values are $(-\pi+\nu)$ or $(-\pi)$. According to the matching condition $|\Delta| \leq \frac{\pi}{2}$, and because $+|\beta\rangle$ is as appropriate for final state as $-|\beta\rangle$ therefore $\pm|\beta\rangle$ can be reached from $\Omega_{\gamma}^{\prime}$ by means of an overall rotation smaller than $\frac{\pi}{2}$. The number of iteration $l_{M C}$ can be expressed from (53) as

$$
l_{M C}=\frac{\frac{\pi}{2}-\left|\arcsin \left(\sin \left(\frac{\phi}{2}-\Lambda+\Lambda_{\gamma}\right) \sin \left(\Omega_{\gamma}\right)\right)\right|}{\Delta},
$$

which leads to a sure success measurement.

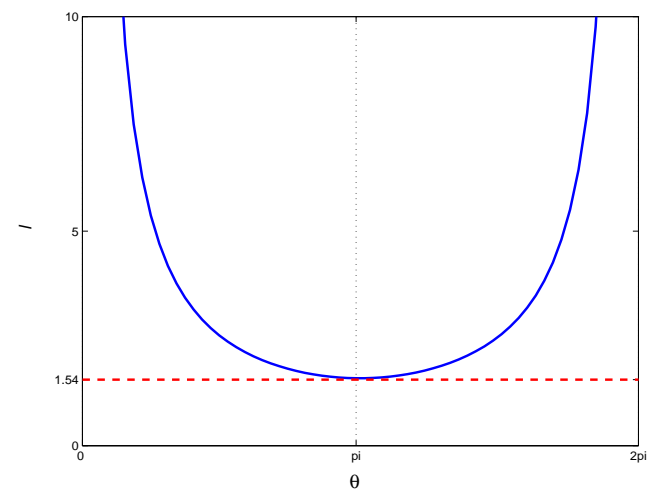

FiguRE 15. Number of iteration $l$ assuming the matching condition is fulfilled, when the parameter setting was $\Omega=0.5$, $\Omega_{\gamma}=0.0001, \Lambda_{\gamma}=0.005, \Lambda=0.005$

4.3.3. The Final State of the Index Register Before the Measurement. An important case could arise when the output of the Grover algorithm should be applied as an input to another circuit. It could be helpful to know the final state of the index register without measuring it. If the initial state with a global phase factor is expressed as

$$
|\gamma\rangle=\left[\cos \left(\Omega_{\gamma}\right)|\alpha\rangle+\sin \left(\Omega_{\gamma}\right) e^{\jmath \Lambda_{\gamma}}|\beta\rangle\right] \cdot e^{\jmath \delta}
$$

after $l_{M C}$ iterations

$$
\mathcal{Q}^{l_{M C}}|\gamma\rangle=e^{\jmath \delta} e^{\jmath\left(l_{M C}\left(\pi+\frac{\phi+\Theta}{2}+\varepsilon\right)\right)}|\beta\rangle,
$$

where

$$
(-1)^{l_{M C}}=e^{\jmath \pi \cdot l_{M C}} .
$$


and

$$
\varepsilon=\arctan \left(\cot \left(\frac{\phi}{2}-\Lambda+\Lambda_{\gamma}\right)\right) .
$$

4.3.4. Possible Variable Settings According to $l_{M C}$. The required number of generalized Grover iteration $l_{M C}$ in (55) refined by the matching condition is typically not an integer. Hence, several cases are worthwhile to discuss how to employ the generalized Grover operator $(\mathcal{Q})$ in order to provide sure success.

(A) In this case the variables $|\gamma\rangle,|\mu\rangle, \phi$ and $\theta$ are predefined. Unfortunately, this fact does not guarantee sure success. A subtask of this assumption is if the settings are $\theta=\phi=\pi$ and $\Lambda=\Lambda_{\gamma}$ as well as $\Omega=\Omega_{\gamma} \Rightarrow|\gamma\rangle=|\mu\rangle$, which leads to the original Grover operator, introduced in Section 2.1.

(B) In the second case let us suppose freely adjustable rotation angles $\phi$ and $\theta$. The optimal $\theta_{\text {opt }}$ is computable from $\frac{\partial l_{M C}}{\partial \theta}=\frac{\partial l_{M C}}{\partial \phi} \cdot \frac{d \phi}{d \theta}+\frac{\partial l_{M C}}{\partial \theta}=0$, i.e. we determine the minimum point of $l_{M C}$ in Fig. 15, from which the optimal rotation angle $\phi_{\text {opt }}$ can be derived applying the matching condition (50), and henceforth the optimal number of iterations $l_{\text {opt }}$ may be find out according to (55), respectively. Since $l$ is not an integer for sure, the - to the next nearest superior positive integer- rounded up $L_{\text {opt }}$ should be taken into account. In consequence of this deferral, the matching condition is harmed, which infer a calibration of the angle $\theta$ and $\phi$. From $L_{o p t_{1}}$ we can calculate $\phi_{o p t_{1}}^{\prime}$ from (55) and substituting it into (50) we get $\theta_{o p t_{1}}^{\prime}$.

(C) In our last case we strive for a single rotation step to the solution. For this purpose only the initial state $|\gamma\rangle$, thus also $\Omega_{\gamma}$ and $\Lambda_{\gamma}$ is given antecedently, whereas all the other parameters are freely customizable.

Starting form equation (55), with $L_{M C}=1$

$$
\Delta=\frac{\pi}{2}-\arcsin \left(\sin \left(\frac{\phi}{2}-\Lambda+\Lambda_{\gamma}\right) \sin \left(\Omega_{\gamma}\right)\right) .
$$

In addition taking the cosine of both sides and substituting $\cos (\Delta)$ form (42) leads to

$$
\tan \left(\frac{\phi}{2}\right)=\frac{\sin \left(\Omega_{\gamma}\right) \sin \left(\Lambda_{\gamma}-\Lambda\right)-\cos \left(\frac{\theta}{2}\right)}{\sin \left(\frac{\theta}{2}\right) \cos (2 \Omega)-\cos \left(\Lambda_{\gamma}-\Lambda\right) \sin \left(\Omega_{\gamma}\right)}
$$

after some trigonometrical calculus. In order to fulfil the matching condition expression (56) should be set equal to $\tan \left(\frac{\phi}{2}\right)$ in (50) by tuning the variables $\Omega, \Lambda$ and $\theta$. This method corresponds to the following visualization: for a given initial state $|\gamma\rangle$ the reflection axis $|\mu\rangle$ is tuned in the vector space $V$ in such a way that it leads to a single rotation to $e^{\jmath \delta} e^{\jmath\left(\pi+\frac{\phi+\theta}{2}+\varepsilon\right)}$, resulting in a solution with a probability of one after a measurement. It should be emphasized that in the original Grover algorithm $|\mu\rangle=|\gamma\rangle$ is used.

\section{EXAmple}

(1) In this example let us have a look at the possible setting of variables in the generalized Grover's search algorithm introduced in Section 4.3.4-C. We examine the algorithm by setting the variables $\Lambda=\Lambda_{\gamma}$ and $\theta=\phi=$ $\pi$ resulting almost in the original Grover operator $(\mathcal{G})$, except that the 
reflection axis $|\mu\rangle$ of the inversion about average operator differs from $|\gamma\rangle$. From equations (50) and (56)

$$
\begin{gathered}
\frac{\cos (2 \Omega)+\sin (2 \Omega) \tan \left(\Omega_{\gamma}\right)}{\cot \left(\frac{\theta}{2}\right)}=\frac{\cos \left(\frac{\theta}{2}\right)}{\sin \left(\Omega_{\gamma}\right)-\sin \left(\frac{\theta}{2}\right) \cos (2 \Omega)}, \\
\cos (2 \Omega)+\sin (2 \Omega) \tan \left(\Omega_{\gamma}\right)=0,
\end{gathered}
$$

resulting in

$$
\Omega=\frac{\frac{\pi}{2}-\Omega_{\gamma}}{2},
$$

which meets our requirement $L_{\text {opt }}=1$.

Finally we emphasize that the definition of $\Omega_{\gamma}$ is different in case of basic and the generalized Grover algorithm. In the former case $\Omega_{\gamma}$ denotes the double of the angle between $|\gamma\rangle$ and $|\alpha\rangle$. In the latter case $\Omega_{\gamma}$ refers to the angle between $|\gamma\rangle$ and $|\alpha\rangle$.

\section{Conclusions}

In this paper we introduced a new generalized Grover operator description applied in quantum database search algorithm. We reviewed the basic Grover database search algorithm and introduced the general Grover operator. We also showed that it is possible to perform a quantum database search using only a single iteration with possibility of success equal one after a measurement.

\section{REFERENCES}

[1] L.K. Grover, "A fast quantum mechanical algorithm for database search," Proceedings, 28th Annual ACM Symposium on the Theory of Computing, pp. 212-219, May 1996. quant$\mathrm{ph} / 9605043$.

[2] L. K. Grover, "Quantum mechanics helps in searching for a needle in a haystack," Phys. Rev. Lett., vol. 79, pp. 325-328, July 1997. e-print quant-ph/9706033.

[3] C. Zalka, "Grover's quantum searching algorithm is optimal," quant-ph/9711070v2, December 1999.

[4] M. Boyer, G. Brassard, P. Hoyer, A. Tapp, "Tight bounds on quantum searching," Proceedings 4th Workshop on Physics and Computation, vol. 46, no. 4-5, pp. 36-43, 1996. Also in Fortschritte der Physik, Vol. 46, No. 4-5, 1998, pp. 493-505 quant-ph/9605034.

[5] C. H. Bennett, E. Bernstein, G. Brassard, U. Vazirani, "Strengths and weakness of quantum computing," SIAM Journal on Computing, vol. 26, no. 5, pp. 1510-1523, 1997. e-print quant$\mathrm{ph} / 9701001$.

[6] C. Zalka, "Simulating quantum systems on a quantum computer," Phys. Rev. A., pp. 313322, 1998.

[7] D. Aharonov, "Quantum computation," Annual Reviews of Computational Physics, vol. VI., 1999. e-print http://www.cs.huji.ac.il/ doria/papers.html.

[8] L. K. Grover, "Quantum computers can search rapidly by using almost any transformation," Phys. Rev. Lett, vol. 80, no. 19, pp. 4329-4332, 1998. e-print quant-ph/9712011.

[9] G. L. Long, Y. S. Li, W. L. Zang and L. Niu, "Phase matching in quantum searching," Phys. Lett., vol. A 262, no. 27, pp. 27-34, 1999. e-print quant-ph/9906020.

[10] G. Brassard, P. Hoyer, M. Mosca, A. Tapp, "Quantum amplitude amplification and estimation," Quantum Computation \& Quantum Information Science, AMS Contemporary Math Series, e-print quant-ph/0005055.

[11] P. Hoyer, "Arbitrary phases in quantum amplitude amplification," Phys. Lett., vol. A 62, 2000. e-print quant-ph/0006031.

[12] G. L. Long, C. C. Tu, Y. S. Li, W. L. Zang and L. Niu, "A novel so(3) picture for quantum searching," Phys. Lett., 1999. e-print quant-ph/9911004.

[13] G. L. Long, "Grover algorithm with zero theoretical failure rate," 2001. e-print quant$\mathrm{ph} / 0106071$. 
[14] G. L. Long, L. Xiao, Y. Sun, "General phase matching condition for quantum searching," 2001. e-print quant-ph/0107013.

[15] J-Y. Hsieh, C-M. Li, "A general su(2) formulation for quantum searching with certainity," 2001. e-print quant-ph/0112035.

[16] D. Biron, O. Biham, E. Biham, M. Grassl, D.A. Lidar, Generalized Grover Search Algorithm for Arbitrary Initial Amplitude Distribution. Lecture Notes in Computer Science, Springer, 1998. Proceedings of the 1st NASA International Conference on Quantum Computing and Quantum Communications, e-print quant-ph/9801066.

[17] E. Biham, O. Biham, D. Biron , M. Grassl, D.A. Lidar, Grover's Search Algorithm for an Arbitrary Initial Amplitude Distribution, vol. 1509 of Lecture Notes in Computer Science, pp. 140-147. Springer, 1998. Quantum Computing \& Quantum Communications; First NASA International Conference; selected papers, QCQC'98, C.P. Williams (ed.), e-print quant$\mathrm{ph} / 9807027$.

[18] E. Biham, O. Biham, D. Biron, M. Grassl, D.A. Lidar, D. Shapira, "Analysis of generalized grover's search algoritms using recursion equations," Phys. Rev., vol. A 63, no. 012310, 2001. e-print quant-ph/00100077.

[19] G. Brassard, P. Hoyer, A. Tapp, Quantum Counting, vol. 1443 of Lecture Notes in Computer Science, pp. 820-831. Springer, July 1998. Proceedings of the 25th International Colloquium on Automata, Languages, and Programming, e-print quant-ph/9805082.

[20] C. Dürr, P. Hoyer, "A quantum algorithm for finding the minimum," 1996. e-print quant$\mathrm{ph} / 9607014$.

[21] A. Ahuja, S. Kapoor, "A quantum algorithm for finding the maximum," e-print quant$\mathrm{ph} / 9911082$.

[22] S. Imre, F. Balázs, Quantum Multi-User Detection in" Application E Services in Wireless Networks", pp. 126-133. Innovative Technology Series, Hermes Penton Science, 2001. ISBN: 1-9039-9630-9.

[23] S. Imre, F. Balázs, "Non-coherent multi-user detection based on quantum search," IEEE International Conference on Communication (ICC), 2002.

[24] S. Imre, F. Balázs, "A tight bound for probability of error for quantum counting based multiuser detection," IEEE International Symposium on Information Theory (ISIT'02), p. 43, Juni 30- July 52002.

[25] L. Grover, "Tradeoffs in the quantum search algorithm," 2002. e-print quant-ph/0201152.

\section{Appendix A. Eigenvalues of the generalized Grover operator $(\mathcal{Q})$}

First, we give a detailed derivation of the eigenvalues of the generalized Grover's search algorithm solving the characteristic function $\operatorname{det}\{\mathbf{Q}-q \mathbf{I}\}$, which is a quiet hard task.

$$
q_{1,2}=\frac{\left(Q_{11}-q\right)\left(Q_{22}-q\right)-Q_{12} Q_{21}=0,}{2},
$$

With the assumption of the basis independent product of eigenvalues with the form of $\operatorname{det}\{\mathbf{Q}\}=q_{1} q_{2}$ as well as with the form of eigenvalues of unitary operators $e^{\jmath \varepsilon}$,

$$
\begin{array}{r}
Q_{11} Q_{22}=(-1)(-1)\left[1+\left(e^{\jmath \theta}-1\right) \cos ^{2}(\Omega)\right] e^{\jmath \phi}\left[1+\left(e^{\jmath \theta}-1\right) \sin ^{2}(\Omega)\right] \\
=e^{\jmath \phi}[1+\left(e^{\jmath \theta}-1\right) \underbrace{\left(\sin ^{2}(\Omega)+\cos ^{2}(\Omega)\right)}_{\equiv 1}+\left(e^{\jmath \theta}-1\right)^{2} \sin ^{2}(\Omega) \cos ^{2}(\Omega)] \\
=e^{\jmath \phi}\left[\left(e^{\jmath \theta}-1\right)^{2} \sin ^{2}(\Omega) \cos ^{2}(\Omega)\right] .
\end{array}
$$




$$
\begin{aligned}
Q_{12} Q_{21}= & (-1)(-1) e^{\jmath \phi}\left(e^{\jmath \theta}-1\right) \sin (\Omega) \cos (\Omega) e^{\jmath \Lambda}\left(e^{\jmath \theta}-1\right) \\
& \sin (\Omega) \cos (\Omega) e^{-\jmath \Lambda}= \\
= & e^{\jmath \phi}\left[\left(e^{\jmath \theta}-1\right) \sin ^{2}(\Omega) \cos ^{2}(\Omega)\right] .
\end{aligned}
$$

Substituting (59) and (60) in (58),

$$
\operatorname{det}(\mathbf{Q})=e^{\jmath(\theta+\phi)}
$$

since $q_{i}=e^{\jmath \varepsilon_{i}}$, by which the eigenvalues of the generalized Grover operator are

$$
q_{1,2}=-e^{\jmath\left(\frac{\theta+\phi}{2} \pm \Delta\right)}
$$

Furthermore, it is known that the trace of $\mathbf{Q}$ can be expressed as

$$
Q_{11}+Q_{22}=q_{1}+q_{2}
$$

resulting in

$$
\begin{array}{r}
Q_{11}+Q_{22}= \\
=-\left[1+\left(e^{\jmath \theta}-1\right) \cos ^{2}(\Omega)+e^{\jmath \phi}\left(e^{\jmath \theta}-1\right) \sin ^{2}(\Omega)\right] \\
=-[\underbrace{1-\cos ^{2}(\Omega)}_{\sin ^{2}(\Omega)}+e^{\jmath \theta} \underbrace{\cos ^{2}(\Omega)}_{1-\sin ^{2}(\Omega)}+e^{\jmath \phi}+ \\
\left.+e^{\jmath(\phi+\theta)} \sin ^{2}(\Omega)-e^{\jmath \phi} \sin ^{2}(\Omega)\right]= \\
=-\left[\sin ^{2}(\Omega)+e^{\jmath \theta}+e^{\jmath \phi}-\sin ^{2}(\Omega)\left(-e^{\jmath \theta}-e^{\jmath \phi}+e^{\jmath(\phi+\theta)}\right)\right],
\end{array}
$$

where the equality stands if both the real and the imaginary part of (64) holds separately. The imaginary one looks like

$$
\begin{array}{r}
\Im\left\{Q_{11}+Q_{22}\right\}= \\
=-\left[\sin (\theta)+\sin (\phi)+\sin ^{2}(\Omega)(-\sin (\theta)-\sin (\phi)+\sin (\phi+\theta))\right]= \\
=-\left\{2 \sin \left(\frac{\phi+\theta}{2}\right) \cos \left(\frac{\phi-\theta}{2}\right)+\right. \\
\left.+\sin ^{2}(\Omega)\left[\sin \left(\frac{\phi+\theta}{2} \cos \left(\frac{\phi-\theta}{2}\right)\right)+2 \sin \left(\frac{\phi+\theta}{2}\right)\right]\right\},
\end{array}
$$

where the trigonometrical definition $\left[\sin x+\sin y=2 \sin \left(\frac{x+y}{2}\right) \cos \left(\frac{x+y}{2}\right)\right]$ is employed. Applying (62) on (63) and substituting into (64),

$$
\begin{aligned}
\Im\left\{q_{1}+q_{2}\right\} & =-\left\{\sin \left(\frac{\theta+\phi}{2}+\Delta\right)+\sin \left(\frac{\theta+\phi}{2}-\Delta\right)\right\} \\
& =-2 \sin \left(\frac{\theta+\phi}{2}\right) \cos (\Delta) .
\end{aligned}
$$


From (65) and (66),

$$
\begin{aligned}
\cos \Delta & =\cos \left(\frac{\phi-\theta}{2}\right)+\sin ^{2}(\Omega)\left(\cos \left(\frac{\theta+\phi}{2}\right)-\cos \left(\frac{\phi-\theta}{2}\right)\right) \\
& =\cos \left(\frac{\phi-\theta}{2}\right)-2 \sin ^{2}(\Omega) \sin \left(\frac{\phi}{2}\right) \sin \left(\frac{\theta}{2}\right) \\
& =\cos \left(\frac{\phi}{2}\right) \cos \left(\frac{\theta}{2}\right)+\sin \left(\frac{\phi}{2}\right) \sin \left(\frac{\theta}{2}\right)\left[1-2 \sin ^{2}(\Omega)\right] \\
& =\cos \left(\frac{\phi}{2}\right) \cos \left(\frac{\theta}{2}\right)+\sin \left(\frac{\phi}{2}\right) \sin \left(\frac{\theta}{2}\right) \cos (2 \Omega) .
\end{aligned}
$$

The derivation of the real part of (64) is straightforward, hence,

$$
\begin{array}{r}
\Re\left\{Q_{11}+Q_{22}\right\}= \\
-\left[2 \cos \left(\frac{\theta+\phi}{2}\right) \cos \left(\frac{\theta-\phi}{2}\right)+\sin ^{2}(\Omega) \cdot 2 \cos ^{2}\left(\frac{\theta+\phi}{2}\right)\right],
\end{array}
$$

thus

$$
\Re\left\{q_{1}+q_{2}\right\}=-2 \cos \left(\frac{\theta+\phi}{2}\right) \cos (\Delta),
$$

whereas we reached the same result as in (67)

$$
\cos \Delta=\cos \left(\frac{\theta-\phi}{2}\right)+\sin ^{2}(\Omega)\left(\cos \left(\frac{\theta+\phi}{2}\right)-\cos \left(\frac{\theta-\phi}{2}\right)\right),
$$

as it was shown in (42).

Consequently, only one restriction is noticeable, namely on $\cos \Delta$, which is $\cos \Delta=\cos (-\Delta)$. At the same time according to the special form of the eigenvalues in (62) the two $\Delta$ 's are equivalent to each other, since both lead to the same eigenvalue pair.

\section{Appendix B. Eigenvectors of the Generalized Grover's SEARch ALGORITHM}

In possession of knowledge about $\mathbf{Q}$ and the eigenvalues $q_{1,2}$, derived above in (62), also the eigenvectors should be found out to the full description of the spectral decomposition of $\mathbf{Q}$.

Starting form (40) and the expression

$$
\left|\psi_{1}\right\rangle=\psi_{1 \alpha}|\alpha\rangle+\psi_{1 \beta}|\beta\rangle
$$

a homogenous linear equation system is obtained

$$
\begin{aligned}
& Q_{11} \psi_{1 \alpha}+Q_{12} \psi_{1 \beta}=q_{1} \psi_{1 \alpha}, \\
& Q_{21} \psi_{1 \alpha}+Q_{22} \psi_{1 \beta}=q_{2} \psi_{1 \beta},
\end{aligned}
$$

from which

$$
\begin{aligned}
\frac{\psi_{1 \alpha}}{\psi_{1 \beta}} & =\frac{q_{1}-Q_{22}}{Q_{21}}, \\
\frac{\psi_{1 \beta}}{\psi_{1 \alpha}} & =\frac{q_{1}-Q_{11}}{Q_{12}} .
\end{aligned}
$$


Apparently, there are infinite many solution of (71), which difference in a scalar factor. For our purposes we only need those with a unit length in a form

$$
|\psi\rangle_{\text {norm }}=\cos (z) e^{\jmath C}|\alpha\rangle+\sin (z)|\beta\rangle
$$

According to (72) let $\psi_{1 \alpha}=q_{1}-Q_{22}$ and $\psi_{1 \beta}=Q_{22}$. From the possible solutions we focus our attention on those, which has unit length, $\|\left|\psi_{1}\right\rangle_{\text {norm }} \|=1$, thus, $\left|\cos (z) e^{\jmath C}\right|^{2}+|\sin (z)|^{2}=1$, where

$$
\begin{aligned}
& \sin ^{2}(z)=\frac{\left|\psi_{1 \beta}\right|^{2}}{\left|\psi_{1 \alpha}\right|^{2}+\left|\psi_{1 \beta}\right|^{2}} \\
& \cos ^{2}(z)=\frac{\left|\psi_{1 \alpha}\right|^{2}}{\left|\psi_{1 \alpha}\right|^{2}+\left|\psi_{1 \beta}\right|^{2}}
\end{aligned}
$$

Following our antecedent establishments

$$
\begin{aligned}
\left|\psi_{1 \alpha}\right|^{2} & =\left|q_{1}-Q_{22}\right|^{2}= \\
& =\overbrace{\left(-\cos \left(\frac{\theta+\phi}{2}+\Delta\right)+\sin ^{2}(\Omega) \cos \left(\frac{\theta+\phi}{2}\right)+\cos ^{2}(\Omega) \cos (\phi)\right)^{2}}^{\Re()}+ \\
& +\underbrace{\left(-\sin \left(\frac{\theta+\phi}{2}+\Delta\right)+\sin ^{2}(\Omega) \sin \left(\frac{\theta+\phi}{2}\right)+\cos ^{2}(\Omega) \sin (\phi)\right)^{2}}_{\Im()},
\end{aligned}
$$

and

$$
\left|\psi_{1 \alpha}\right|^{2}=\psi_{1 \alpha} \psi_{1 \alpha}^{*},
$$

$$
\left|\psi_{1 \beta}\right|^{2}=\psi_{1 \beta} \psi_{1 \beta}^{*},
$$


respectively. As the next step let us derive $\left|\psi_{1 \alpha} / \psi_{1 \beta}\right|^{2}$ as follows

$$
\begin{aligned}
& \left|\frac{\psi_{1 \alpha}}{\psi_{1 \beta}}\right|^{2}=\frac{-e^{\jmath\left(\frac{\theta+\phi}{2}+\Delta\right)}+e^{\jmath \phi}\left[\left(e^{\jmath \theta}-1\right) \sin ^{2}(\Omega)+1\right]}{-e^{\jmath \phi}\left(e^{\jmath \theta}-1\right) \sin (\Omega) \cos (\Omega) e^{-\jmath \Lambda}} . \\
& \frac{-e^{-\jmath\left(\frac{\theta+\phi}{2}+\Delta\right)}+e^{-\jmath \phi}\left[\left(e^{-\jmath \theta}-1\right) \sin ^{2}(\Omega)+1\right]}{-e^{-\jmath \phi}\left(e^{-\jmath \theta}-1\right) \sin (\Omega) \cos (\Omega) e^{\jmath \Lambda}} \\
& =\frac{\left(1-e^{\jmath\left(\frac{\theta-\phi}{2}+\Delta\right)}+\left(e^{\jmath \theta}-1\right) \sin ^{2}(\Omega)\right)}{\left(e^{\jmath \theta}-1\right)\left(e^{-\jmath \theta}-1\right) \sin ^{2}(\Omega) \cos ^{2}(\Omega)} . \\
& \frac{\left(1-e^{-\jmath\left(\frac{\theta-\phi}{2}+\Delta\right)}+\left(e^{-\jmath \theta}-1\right) \sin ^{2}(\Omega)\right)}{\left(e^{\jmath \theta}-1\right)\left(e^{-\jmath \theta}-1\right) \sin ^{2}(\Omega) \cos ^{2}(\Omega)} \\
& =\frac{\left[1-e^{\jmath\left(\frac{\theta-\phi}{2}+\Delta\right)}-e^{-\jmath\left(\frac{\theta-\phi}{2}+\Delta\right)}+1\right]+\left[1-e^{-\jmath \theta}-e^{\jmath \theta}+1\right] \sin ^{4}(\Omega)}{\sin ^{2}(\Omega) \cos ^{2}(\Omega)\left[1-e^{-\jmath \theta}-e^{\jmath \theta}+1\right]}+ \\
& +\frac{\sin ^{2}(\Omega)\left[e^{\jmath \theta}-1-e^{\jmath\left(\frac{\theta+\phi}{2}-\Delta\right)}+e^{-\jmath\left(\frac{\theta-\phi}{2}+\Delta\right)}+e^{-\jmath \theta}-1-e^{-\jmath\left(\frac{\theta+\phi}{2}-\Delta\right)}+e^{\jmath\left(\frac{\theta-\phi}{2}+\Delta\right)}\right]}{\sin ^{2}(\Omega) \cos ^{2}(\Omega)\left[1-e^{-\jmath \theta}-e^{\jmath \theta}+1\right]}= \\
& =\frac{2-2 \cos \left(\frac{\theta-\phi}{2}+\Delta\right)-\sin ^{2}(\Omega) \cos ^{2}(\Omega)[2-2 \cos (\theta)]}{\sin ^{2}(\Omega) \cos ^{2}(\Omega)[2-2 \cos (\theta)]}+ \\
& +\frac{\sin ^{2}(\Omega)\left[2-2 \cos (\theta)-2+2 \cos (\theta)-2 \cos \left(\frac{\theta+\phi}{2}-\Delta\right)+2 \cos \left(\frac{\theta-\phi}{2}+\Delta\right)\right]}{\sin ^{2}(\Omega) \cos ^{2}(\Omega)[2-2 \cos (\theta)]}= \\
& =\frac{2-2 \cos \left(\frac{\theta-\phi}{2}+\Delta\right)-\overbrace{\sin ^{2}(\Omega) \cos ^{2}(\Omega) 4}^{\sin ^{2}(2 \Omega)} \sin ^{2}\left(\frac{\theta}{2}\right)}{\sin ^{2}(2 \Omega) \sin ^{2}\left(\frac{\theta}{2}\right)}+ \\
& +(8 \theta) \frac{\sin ^{2}(\Omega)\left[2 \cos \left(\frac{\theta-\phi}{2}+\Delta\right)-2 \cos \left(\frac{\theta+\phi}{2}-\Delta\right)\right]}{\sin ^{2}(2 \Omega) \sin ^{2}\left(\frac{\theta}{2}\right)}
\end{aligned}
$$

Keeping in mind the expression (75), in which $\left|\psi_{1 \alpha} / \psi_{1 \beta}\right|^{2}$ is given in (80),

$$
\begin{aligned}
\frac{\left|\psi_{1 \beta}\right|^{2}}{\left|\psi_{1 \alpha}\right|^{2}+\left|\psi_{1 \beta}\right|^{2}} & =\frac{\sin ^{2}(2 \Omega) \sin ^{2}\left(\frac{\theta}{2}\right)}{2-2 \cos \left(\frac{\theta-\phi}{2}+\Delta\right) \sin ^{2}(\Omega)\left[2 \cos \left(\frac{\theta-\phi}{2}+\Delta\right)-2 \cos \left(\frac{\theta+\phi}{2}-\Delta\right)\right]} \\
& =\frac{\sin ^{2}(2 \Omega) \sin ^{2}\left(\frac{\theta}{2}\right)}{2-2 \cos \left(\frac{\theta-\phi}{2}+\Delta\right)+4 \sin ^{2}(\Omega) \sin \left(\frac{\theta}{2}\right) \sin \left(\frac{\phi}{2}-\Delta\right)} \\
& =\frac{\sin ^{2}(2 \Omega) \sin ^{2}\left(\frac{\theta}{2}\right)}{2-2 \cos \left(\frac{\theta}{2}\right) \cos \left(\frac{\phi}{2}-\Delta\right)-\underbrace{2 \sin \left(\frac{\theta}{2}\right) \sin \left(\frac{\phi}{2}-\Delta\right)+4 \sin ^{2}(\Omega) \sin \left(\frac{\theta}{2}\right) \sin \left(\frac{\phi}{2}-\Delta\right)}_{\sin \left(\frac{\theta}{2}\right) \sin \left(\frac{\phi}{2}-\Delta\right)}}
\end{aligned}
$$

(81) $\sin ^{2}(z)=\frac{\sin ^{2}(2 \Omega) \sin ^{2}\left(\frac{\theta}{2}\right)}{2\left(1-\cos \left(\frac{\theta}{2}\right) \cos \left(\frac{\phi}{2}-\Delta\right)-2 \cos (2 \Omega) \sin \left(\frac{\theta}{2}\right) \sin \left(\frac{\phi}{2}-\Delta\right)\right)}$.

$$
\cos ^{2}(z)=1-\sin ^{2}(z)
$$

Finally, to determine the eigenvectors $\left|\psi_{1,2}\right\rangle$, only $e^{\jmath C}$ part is remaining in (74). Considering the relation

$$
\frac{\psi_{1 \alpha}}{\psi_{1 \beta}}=\frac{\cos (z)}{\sin (z)} e^{\jmath C_{1}}
$$


and thus

$$
\left|\frac{\psi_{1 \alpha}}{\psi_{1 \beta}}\right|^{2}=\cot ^{2}(z) e^{\jmath 2 C_{1}}=\frac{Q_{12}}{Q_{21}} \cdot \frac{q_{1}-Q_{22}}{q_{1}-Q_{11}},
$$

where equations (72), (73) were employed, and

$$
\cot (2 z)=\frac{\cos ^{2}(z)-\sin ^{2}(z)}{2 \sqrt{\sin ^{2}(z) \cos ^{2}(z)}}=\frac{\left|\psi_{1 \alpha}\right|^{2}-\left|\psi_{1 \beta}\right|^{2}}{2 \sqrt{\left|\psi_{1 \alpha}\right|^{2}\left|\psi_{1 \beta}\right|^{2}}},
$$

respectively. It can be proved that

$$
\frac{q_{1}-Q_{22}}{q_{1}-Q_{11}}
$$

is a real number, which implies that

from which follows

$$
\left(e^{\jmath C_{1}}\right)^{2}=\frac{Q_{12}}{Q_{21}}=\frac{e^{-\jmath \Lambda} e^{\jmath \phi}}{e^{-\jmath \Lambda}}=e^{\jmath(\phi-2 \Lambda)},
$$

$$
e^{\jmath C_{1}}= \pm e^{\jmath\left(\frac{\phi}{2}-\Lambda\right)}
$$

Based on (83) the normalized eigenvector

$$
\left|\psi_{1}\right\rangle=\cos (z) e^{\jmath\left(\frac{\phi}{2}-\Lambda\right)}|\alpha\rangle+\sin (z)|\beta\rangle .
$$

Eigenvector $\left|\psi_{2}\right\rangle$ have to be calculated in a similar way, where the other eigenvalue $q_{2}$ should be taken into account, which results in a simple sign change of $\Delta$. Due to the definition of $C_{2}$ in (74), it does not depend on the sign of $\Delta$, thus $e^{\jmath C_{2}}= \pm e^{\jmath C_{1}}$. To become the eigenvectors $\left|\psi_{1}\right\rangle$ and $\left|\psi_{2}\right\rangle$ orthogonal, $e^{\jmath C_{2}}$ must be equal to $-e^{\jmath C_{1}}$, whereas the second eigenvector will be

$$
\left|\psi_{2}\right\rangle=-\sin (z) e^{\jmath\left(\frac{\phi}{2}-\Lambda\right)}|\alpha\rangle+\cos (z)|\beta\rangle
$$

Budapest University of Technology and Economics, Department of Telecommunication, Mobile Communications and Computing Laboratory, H-1117 Budapest, Magyar TUDÓSOK KÖRÚTJA 2., HUNGARY

E-mail address: balazsf@hit.hit.bme.hu 